\title{
Crowd-sourcing world models with OpenRoomMap
}

\author{
Andrew C. Rice and Oliver J. Woodman \\ Computer Laboratory \\ University of Cambridge \\ Cambridge \\ \{acr31,ojw28\}@cam.ac.uk
}

\begin{abstract}
The construction of world models typically requires laborious survey and cataloguing of a building's contents. OpenRoomMap attempts to crowd-source this information by allowing all building occupants to contribute to the model. Our initial deployment of OpenRoomMap has considered our office building that has a floor area of approximately 10000 square meters over 3 floors. Building occupants have mapped approximately $70 \%$ of the building and placed over 4000 objects. We show how the accuracy of placement depends on the physical restrictions that constrain the positions of objects and discuss the strategies we are using to encourage ongoing update and maintenance of the data.
\end{abstract}

Keywords-context-aware, world-model, crowd-sourcing, VGI

\section{INTRODUCTION}

Pervasive Computing has long supported context-aware applications by providing the sensor data that forms the basis of context-sensitive inferences. These inferences are often made with reference to a world model that provides additional contextual information about the application's environment. For example, many of the applications built upon the Active Bat location system [1] rely on such a model provided by the SPIRIT middleware [2]. One such application teleports a user's desktop to a monitor near to their current location. For this application the model must provide information about computers in the environment and the locations of monitors to which they connect.

Constructing world models is a laborious task [3] and keeping them up-to-date requires ongoing effort. The amount of effort required can be reduced by automation to some extent [4] but much manual effort still remains.

As an alternative we have been exploring the possibility for crowd-sourcing the development and maintenance of inbuilding world models. Wikipedia is one of the most prominent examples of the ability of a large community to generate and maintain an enormous repository of knowledge. More recently, the OpenStreetMap project has demonstrated how volunteers can build accurate and detailed maps comparable with commercial counterparts simply through the annotation of data collected through commodity GPS receivers [5]. In Geographic Information Systems (GIS) community there are an increasing number of examples of Volunteer Generated Information (VGI) in the collection and annotation of mapping data [6].

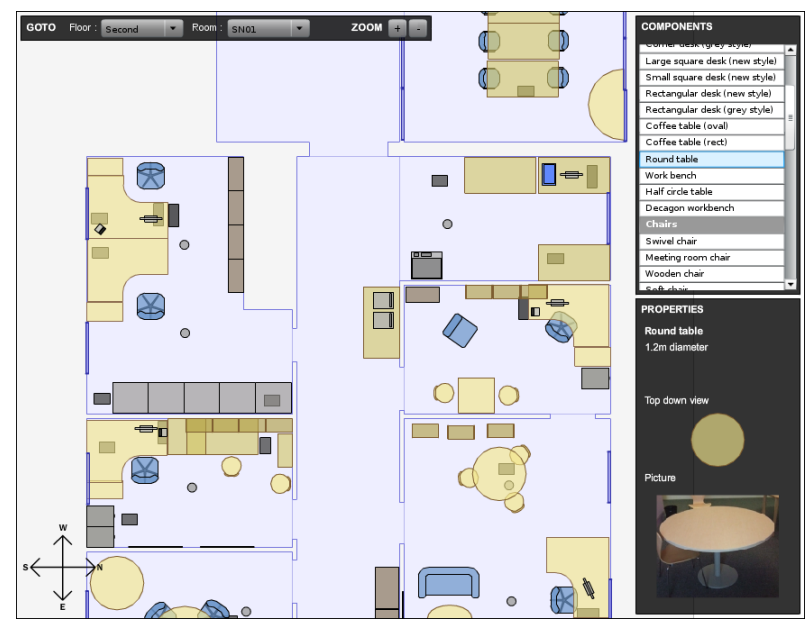

Figure 1. The OpenRoomMap interface provides an editable plan of the building and a toolbox to add new items.

OpenRoomMap is our attempt to apply these same ideas within buildings. In this paper we present our initial implementation and describe the deployment of OpenRoomMap in our own office building. We evaluate the accuracy of user-contributed information against our own surveys and discuss our activities in encouraging ongoing maintenance of the model. We highlight a number of applications that have arisen for OpenRoomMap data and describe our intentions for future work.

\section{OpenRoOMMAP}

Surveying and mapping the locations of furniture and appliances within a large building is a huge effort. OpenRoomMap attempts to alleviate this by distributing the effort between the occupants of the building.

At present an OpenRoomMap model consists of a noneditable floor plan on which instances of defined object types are placed. Object types currently include furniture items such as desks and chairs, electronic equipment including computers, monitors, printers and telephones, as well as data points and air vents. Certain object types also have semantic data fields that can be edited by the user. For example each instance of a telephone has an associated telephone number and each computer has a name. For the sake of simplicity 


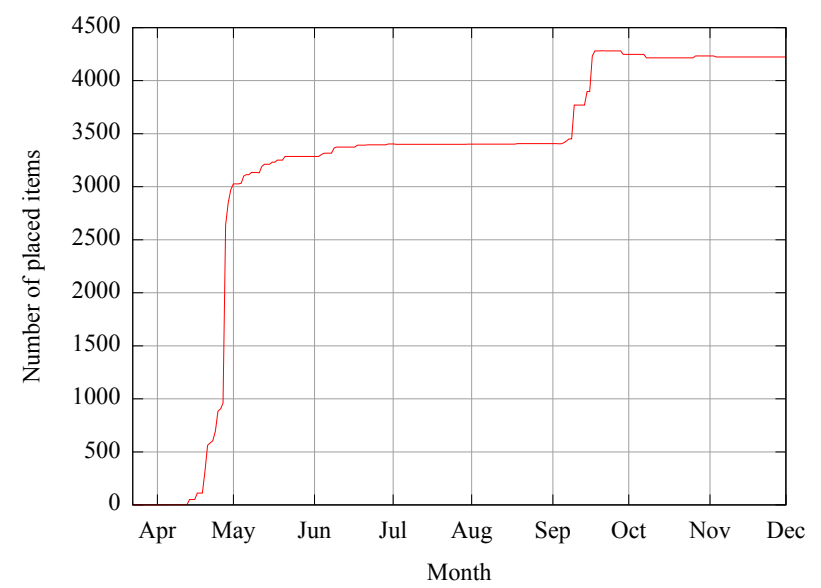

Figure 2. The number of items in the model increased rapidly at the launch and then increased again when further functionality was added.

the current model does not encode relationships between objects. For example a monitor is not explicitly associated with the computer to which it is attached. Nevertheless it is often possible to automatically infer such relationships from the model, for example by considering the distance between two objects.

We developed a web-based editor (Figure 1) to allow building occupants to contribute to the model. Our editor allows users to browse the floor plan and to add, edit and remove instances of the defined object types. All changes to the model are immediately saved and made visible to all other viewers. Our initial implementation places no constraints on the position of any object. The object definitions were created by administrators based on the types of furniture in the building.

\section{PRELIMINARY DEPLOYMENT}

Our first experiment with OpenRoomMap has been to map our own building that comprises around 250 occupants (excluding students) and occupies approximately $10000 \mathrm{~m}^{2}$ over 3 floors. We derived a building plan from manually corrected architectural drawings. We launched the study by email to staff members who placed around 3000 objects over 5 hours. After a period of approximately 6 months the map is now approximately $70 \%$ full with 139 out of 207 rooms completed. Figure 2 shows the number of placed objects against time.

We feel that social effects arising from the fact that our building's occupants form a closed community resulted in different behaviour from users compared to opencommunity collaborative efforts. One particular example was the "whale" (Figure 3). This act of graffiti was created out of furniture over the period of about 30 minutes. However, contrary to examples of defacement in open-community efforts the same individuals subsequently spent a further

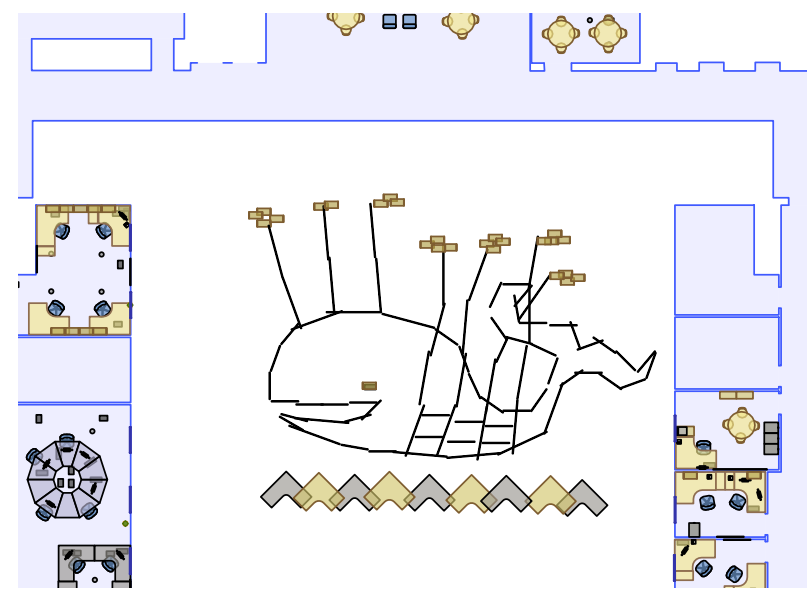

Figure 3. Considerable collaborative activity focused on drawing pictures with furniture.

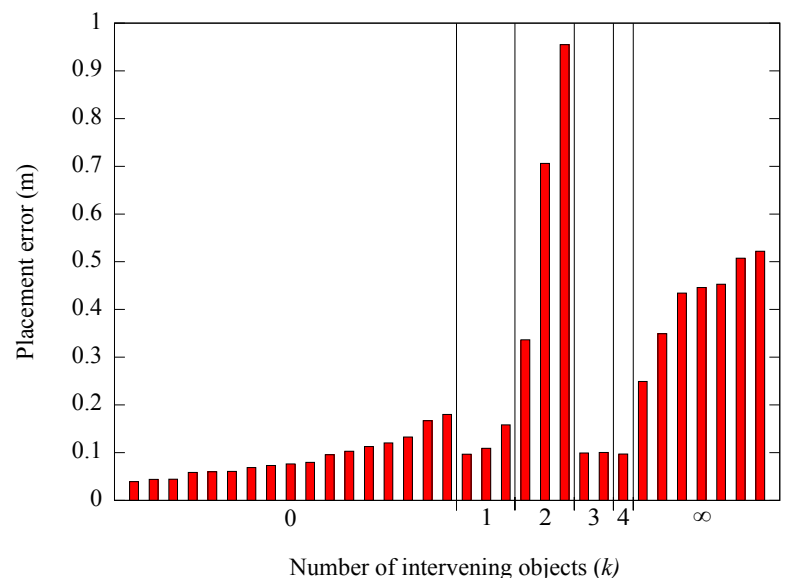

Figure 4. The placement accuracy for $k$-constrained objects. Items that were harder to place (i.e. those with larger $k$ values) were generally positioned less accurately.

30 minutes dismantling it. When asked about this later the artists said that they had decided to take it down again because they didn't want to annoy people.

A misunderstanding of the interface meant that some users took already-placed furniture and moved it into their offices rather than creating new items. In another example of the social effects of a closed-community one member of staff physically walked down the corridor in order to retrieve a virtual desk that had been taken from his office.

\section{Placement accuracy}

We made a preliminary study into the accuracy of object placement by comparing the positions of desks in the OpenRoomMap model with those in the Spirit database used by the Active Bat location system. We have no context-aware applications running in our building that make use of the position of desks and so there is no particular incentive to 


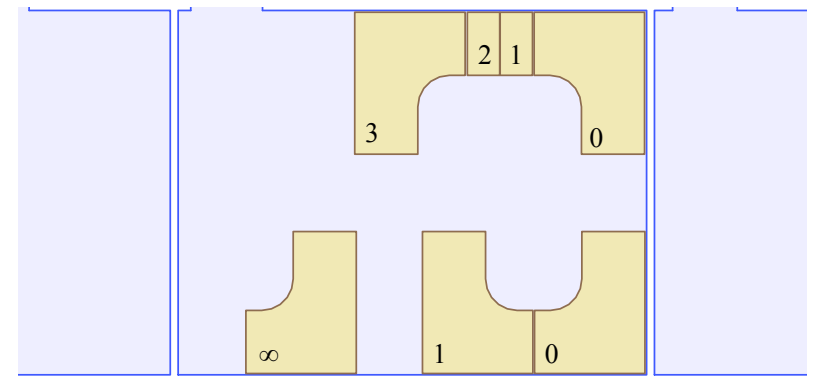

Figure 5. An office containing $k$-constrained objects of differing degree $k$. Each object is marked with its degree.

occupants for accurately positioning them over and above a visual reward in the building map. Nonetheless we see that most objects are well within $20 \mathrm{~cm}$ of their manually surveyed positions, as shown in Figure 4. We also see a correlation between accuracy and the difficulty of placement, which we characterise by the number of intervening objects required to constrain the position of the object to a reference point on the floor plan. We say that an object located in the corner of a room is 0-constrained because no other furniture is required to constrain its $(x, y)$ position. In other words the user can align the corner of the desk directly with the corner of the room when placing that object. If an object can be placed by the same method but by aligning with the corner of a k-constrained object then we say that the object is $(\mathrm{k}+1)$-constrained, as shown in Figure 5. An object free-floating along a wall is said to be $\infty$-constrained because no constraints are available to restrict its position in both the $x$ and $y$ directions. The results shown in Figure 4 suggest that techniques to aid in the visual positioning of $\infty$-constrained objects could be useful for improving accuracy and that improving the precision of $n$-constrained placement though object snapping (for example) might have little overall benefit.

\section{ENCOURAGING UPDATES}

Somewhat predictably the number of edits rapidly declined after an initial surge of interest. To encourage ongoing updates we implemented a number of additional services using the OpenRoomMap data. The principle being that if occupants are using the model regularly then they will have an incentive to provide corrections. We implemented the following services that make use of the OpenRoomMap model:

- A read-only HTML version of the OpenRoomMap data ${ }^{1}$ to serve as an alternative floor-plan for the building;

- Integration with the people directory page of our main website such that clicking on the name of an office shows its location on the floor plan;

\footnotetext{
${ }^{1}$ Built using OpenLayers in the same manner as OpenStreetMap.
}

- Integration with the room bookings pages of our internal website so that the contents and configuration of bookable rooms can be browsed;

- Integration (ongoing) with the typographic resources pages of our internal site to help users find the location of printers and photocopiers.

The deployment of these features produced another set of updates to the building contents (visible as the second step change in Figure 2). The primary instigators of this second round of changes were the building manager and administrative staff who were interested in a particular application. This two phase model of operation seems quite appropriate. In the first phase, in an initial surge of interest, many occupants of the building contribute to a general mapping (much like an OpenStreetMap mapping event). In the second phase, the map moves into maintenance mode and it is only necessary to record changes as they occur. This activity is feasibly within the remit of the building management staff who are often involved in any office reconfiguration anyway.

\section{Applications}

In addition to the obvious use of OpenRoomMap as a world model for context-aware applications, a number of other applications have also arisen:

- Building inventory The managers of our building maintain records of the building inventory in order to track the locations of items such as heaters or desk fans. OpenRoomMap can act as a repository for this information.

- Floor-box location Our network monitoring infrastructure can identify which floor-box in the structured wiring system a particular machine is attached to but we have no records of where in a particular room the floor-boxes are. In our teaching rooms, in particular, this can make identifying a particular machine a tedious process.

- Pedestrian localisation The accuracy of pedestrian localisation systems can be improved by providing knowledge of constraints which limit the movement of a pedestrian through the environment [7]. We have used furniture constraints obtained from OpenRoomMap to improve the performance of one such system.

\section{FUTURE WORK}

Our work so far on OpenRoomMap has been limited to a single building. Our immediate plans are to move on to map additional buildings. Particular shortcomings that need to be addressed are an inability to update the floor plan and also a lack of an interface to define new furniture types ${ }^{2}$.

We are also interested in exploiting closed-community social effects in the editing. One particular feature we would

\footnotetext{
${ }^{2}$ We added "frivolous" items such as pot-plants into our deployment by popular request.
} 
like to try is to visually ascribe edits to particular individuals. This would mean for example, that potential editors might be encouraged by noticing that the Head of Department has entered the contents of his own office.

Ensuring continuing updates to the existing OpenRoomMap data is a key challenge that we hope to approach by continuing to integrate the data-set into building operations. Another example application to support is the visualisation of sensor data. For example, we have data-sets providing data such as location information for individuals, indoor temperature and wireless coverage. Providing access to this information might be another means to encourage updates.

This work is part of the Sense and Optimise theme in Computing for the Future of the Planet [8]. We see OpenRoomMap as a key tool for collecting and managing data about the world with minimal energy consumption and sensing infrastructure.

\section{ACKNOWLEDGEMENT}

Grateful thanks are due to Professor Andy Hopper for his support and contributions to the project and to Dr Alastair Beresford for useful discussions on some of the issues. Thanks to the occupants of the William Gates Building for participating our study and particularly Ian Burton-Palmer for helping will all aspects of the building management information.

\section{REFERENCES}

[1] A. Ward, A. Jones, and A. Hopper, "A new location technique for the active office," IEEE Personal Communications, vol. 4, no. 5 , pp. 42-47, Oct. 1997.

[2] M. Addlesee, R. Curwen, S. Hodges, J. Newman, P. Steggles, A. Ward, and A. Hopper, "Implementing a sentient computing system," Computer, vol. 34, no. 8, pp. 50-56, 2001.

[3] J. Scott and M. Hazas, "User-friendly surveying techniques for location-aware systems," in Proceedings of the Fifth International Conference on Ubiquitous Computing, vol. 2864, Oct. 2003, pp. 45-54.

[4] R. K. Harle, "Maintaining world models in context-aware systems," Ph.D. dissertation, University of Cambridge, 2004.

[5] M. Haklay and P. Weber, "Openstreetmap: User-generated street maps," Pervasive Computing, IEEE, vol. 7, no. 4, pp. 12-18, Oct.-Dec. 2008.

[6] M. Goodchild, "Citizens as sensors: the world of volunteered geography," GeoJournal, vol. 69, no. 4, pp. 211-221, 2007. [Online]. Available: http://dx.doi.org/10.1007/s10708-007-9111-y

[7] O. Woodman and R. Harle, "Pedestrian localisation for indoor environments," in UbiComp '08: Proceedings of the 10th international conference on Ubiquitous computing, 2008, pp. 114-123.
[8] A. Hopper and A. Rice, "Computing for the future of the planet," Philosophical Transactions of the Royal Society A, vol. 366, no. 1881, pp. 3685-3697, 2008. 